\title{
Comparing the Fatigue and Corrosion Behavior of Nanograin and Coarse-Grain IF Steels
}

\author{
A. Chabok $\cdot$ K. Dehghani $\cdot$ M. Ahmadi Jazani
}

Received: 17 February 2014/Revised: 24 June 2014/Published online: 7 January 2015

(C) The Chinese Society for Metals and Springer-Verlag Berlin Heidelberg 2015

\begin{abstract}
In the present work, a nanograin layer of about $150 \mu \mathrm{m}$ thick was formed on the surface of an interstitial-free (IF) steel via friction stir processing. Then, the fatigue and corrosion behaviors of IF steel with nanograin layer were compared with that of coarse-structure counterpart. More than threefold increase in the hardness was observed due to the formation of nanograin layer. The size of nanograins in the stir zone was within 30-150 nm. This resulted in 50\% increase in the fatigue strength of nanostructured specimen. Furthermore, the fracture surfaces were characterized using field emission scanning electron microscopy and scanning electron microscopy. As for the fatigue behavior of nanograin IF steel, the fracture surface was characterized by the formation of nanospacing striations and nanodimples. Besides, the nanograin structure pronounced the passivity and exhibited higher corrosion resistance.
\end{abstract}

KEY WORDS: Nanograin; Interstitial-free (IF) steel; Fatigue; Friction stir processing (FSP); Corrosion

\section{Introduction}

Comparing to coarse-grain structures, nanostructured materials can significantly enhance the resistance to fatigue crack initiation. Thus, a material with a nanostructure layer on its surface can exhibit higher fatigue resistance because of pronounced resistance to crack initiation and slower crack growth rate due to coarse grains at the interior of material [1,2]. A nanostructured layer can be formed on the surface of materials via techniques such as surface mechanical attrition [3, 4], surface nanocrystallization, hardening [5, 6] and wire brushing [7]. Comparing to the conventional methods, friction stir processing (FSP) is a unique approach for surface nanostructuring of materials $[8,9]$. Because of significant structure refinement during

Available online at http://link.springer.com/journal/40195

A. Chabok $(\bowtie) \cdot$ K. Dehghani · M. Ahmadi Jazani

Mining and Metallurgical Engineering Department, Amirkabir

University of Technology, Tehran, Iran

e-mail: ali.chabok@aut.ac.ir
FSP, considerable enhancement in mechanical properties of various materials can be reached [10, 11]. For example, it is reported that FSP technique was used successfully to produce nanograin layers on the surface of interstitial-free (IF) steel [12].

Furthermore, there are many studies [13-17] on the corrosion behavior of nanograin materials compared to their coarse-grain counterparts. In this regard, Hadizma et al. [14] evaluated the corrosion behavior of IF steel processed by ECAP and indicated that with increasing the ECAP passes, which leads to finer grains, the pitting potential increases. Lee et al. [16] showed that nanocrystallized surface of AISI 304SS exhibited higher corrosion resistance than that of coarse-grain surface. It has been reported [18] that $\mathrm{Ti}$ with the grain size of $330 \mathrm{~nm}$ exhibited higher corrosion resistance than that of coarsegrain size. Dehghani et al. [19] clarified that the nanostructured layer produced via wire brushing, reduced the corrosion rate and enhanced the corrosion resistance of IF steel comparing to its coarse-grain structure.

In this study, the fatigue and corrosion behavior of FSPed IF steel were investigated. As IF steels are used 
extensively as car bodies, they are readily exposed to a wide variety of industrial and air pollutions. Thus, it was aimed to focus on the corrosion behavior of this steel having nanostructured surface. Therefore, in terms of atmospheric and industrial environments, the used media were the solutions containing $\mathrm{H}_{2} \mathrm{SO}_{4}$ as a source of $\mathrm{SO}_{2}$ and $\mathrm{NaCl}$ as a source of $\mathrm{Cl}^{-}$ion, respectively.

\section{Experimental}

The used IF steel (Fe-0.002 C-0.003 N-0.424 Ti-0.005 S, in wt $\%$ ) had the thickness of $1.5 \mathrm{~mm}$. The initial grain size of studied material was about $60 \mu \mathrm{m}$. The specimen of $20 \mathrm{~cm} \times 10 \mathrm{~cm}$ was subjected to FSP using a tungstencarbide tool having a $16 \mathrm{~mm}$ diameter shoulder only, thus no pin was used for FSP. As IF steels are widely used as car bodies, the thinnest possible sheet was selected. The rotating and traverse speeds were $1,600 \mathrm{r} / \mathrm{min}$ and $31.5 \mathrm{~mm} / \mathrm{min}$, respectively. The processing parameters were adjusted so that the temperature during FSP was about $(900 \pm 2){ }^{\circ} \mathrm{C}$ to inhibit austenite transformation. A mixture of water and ice was used to quench the workpiece immediately after FSP to prevent any grain growth. Both sides of specimens were subjected to FSP. Then, fatigue samples were cut parallel to the FSP direction according to ASTME-466 with the dimensions shown in Fig. 1. The samples were then subjected to the load-controlled fatigue tests using a Instron-IX machine. The fatigue tests were carried out at the constant stress ratio $(R)$ of zero and the frequency of $3 \mathrm{~Hz}$.

The samples $(1 \mathrm{~cm} \times 1 \mathrm{~cm})$ for corrosion testing were prepared from the IF steel having a nanostructure layer. Different corrosive media were employed to investigate and compare the corrosion behavior of coarse-grain (CG) with nanograin (NG) IF steel. The solutions contained $\mathrm{H}_{2} \mathrm{SO}_{4}$ as a source of $\mathrm{SO}_{2}$ and $\mathrm{NaCl}$ as a source of $\mathrm{Cl}^{-}$ion were used as the corrosive media, respectively. Different used media with the compositions of $3.5 \mathrm{wt} \% \mathrm{NaCl}$ (No. 1), $0.1 \mathrm{~mol} / \mathrm{L} \mathrm{H}_{2} \mathrm{SO}_{4}$ (No. 2), $2.5 \mathrm{wt} \% \mathrm{NaCl}+0.05 \mathrm{~mol} / \mathrm{L}$ $\mathrm{H}_{2} \mathrm{SO}_{4}$ (No. 3) and $3.5 \mathrm{wt} \% \mathrm{NaCl}+0.1 \mathrm{~mol} / \mathrm{L} \mathrm{H}_{2} \mathrm{SO}_{4}$ (No. 4). Corrosion current densities $\left(i_{\text {corr }}\right)$ and corrosion potentials $\left(E_{\text {corr }}\right)$ were then obtained by plotting the individual potentiodynamic curves using the Tafel extrapolation procedure. Microstructural evolutions were investigated using scanning electron microscopy (SEM) and field emission electron microscopy (FESEM).

\section{Results and Discussion}

\subsection{Producing Nanostructure Surface Layer by FSP}

The SEM taken from the cross section of stirred zone is shown in Fig. 2a. Three regions are recognized as: stirred zone, transition zone and the base material. The top layer formed by FSP experiences the severest deformation as well as the highest cooling rate after the FSP. Consequently, the nanograins are formed in this region. The original structure was not affected by the friction stir processing keeping its initial grain size. The transition zone, which is located between the top and central regions, is partially affected by FSP and grains are elongated in this region. Figure $2 \mathrm{~b}$ illustrates the FESEM regarding top

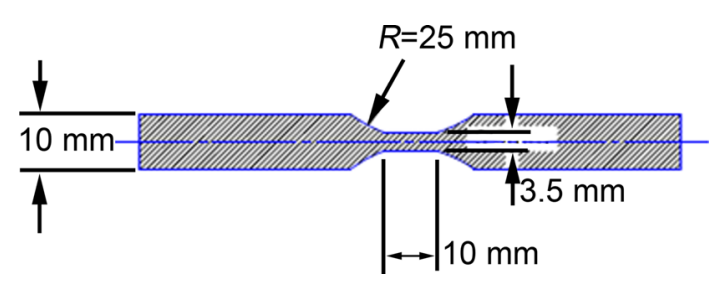

Fig. 1 Schematic and dimensions of the fatigue test specimens
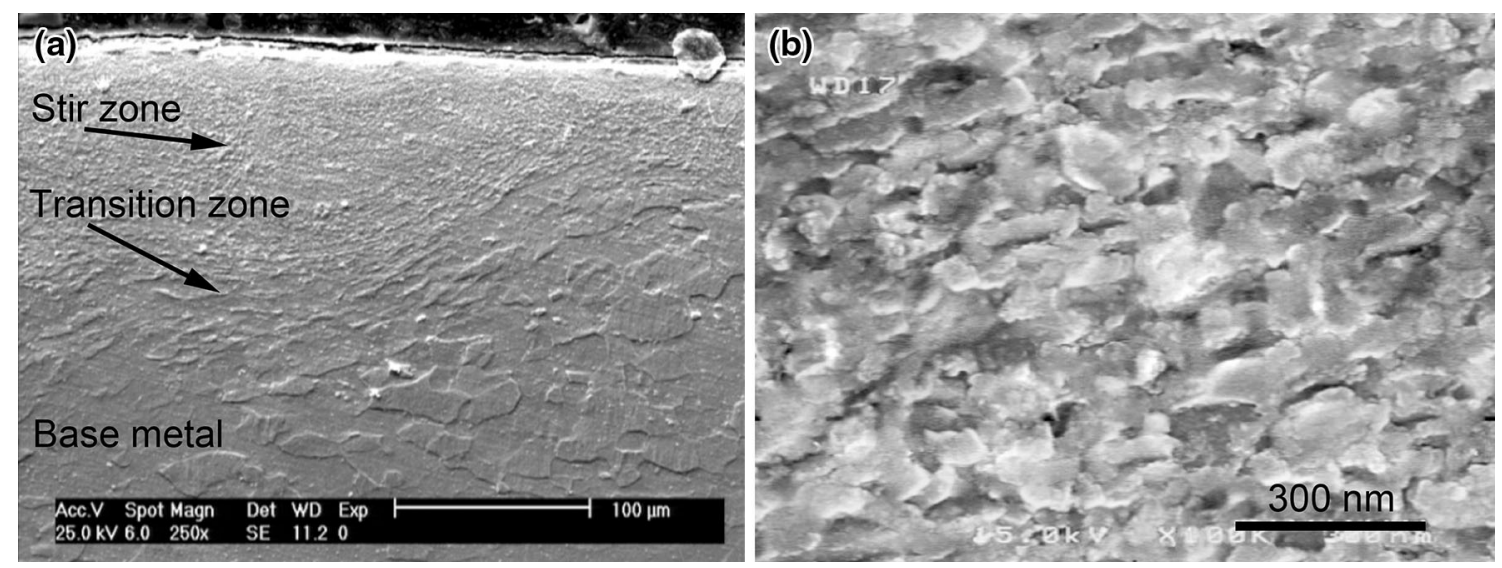

Fig. 2 a SEM taken from cross section of friction stir processed zone, b FESEM confirming the nanograins in stir zone/top surface 


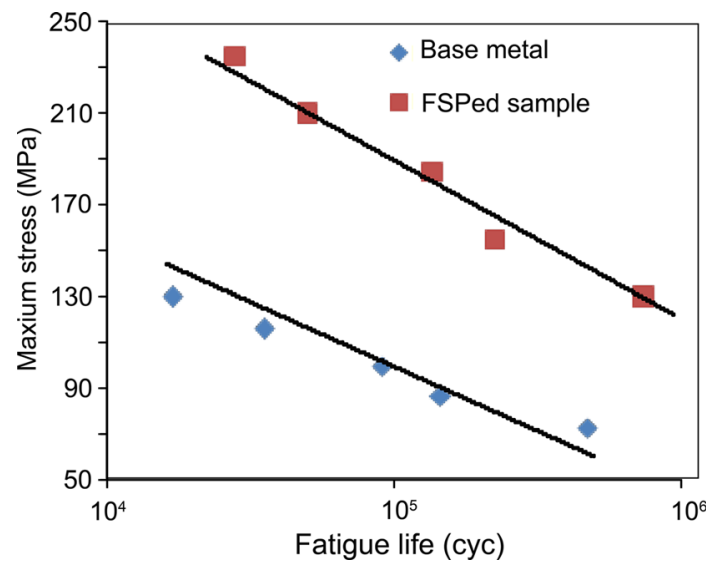

Fig. 3 Stress-life diagrams of as-received and FSPed IF steel

surface or stir zone. According to this figure, the nanograins of $30-150 \mathrm{~nm}$ were formed in the stir zone of processed material.
The thermomechanical treatment (i.e., severe plastic deformation at high temperature) applied to the top surface resulted in the formation of nanograins via dynamic recovery (DRV). DRV is the dominate restoration mechanism during the thermomechanical treatment of high SFE materials such as IF steels. The formation mechanisms of nanograins during FSP of high SFE aluminum alloys and IF steels are discussed elsewhere by Yazdipour et al. [9] and Chabok et al. [12].

The changes in cross-sectional hardness, from the top toward the center of sheet, were measured after FSP. The microhardness of nanostructured layer was about $317 \mathrm{HV}$ and decreased continuously toward the center, reaching to $100 \mathrm{HV}$ pertaining to base metal.

\subsection{Fatigue Behavior}

According to the Hall-Petch equation, the changes in hardness are inversely proportional to the size of grains
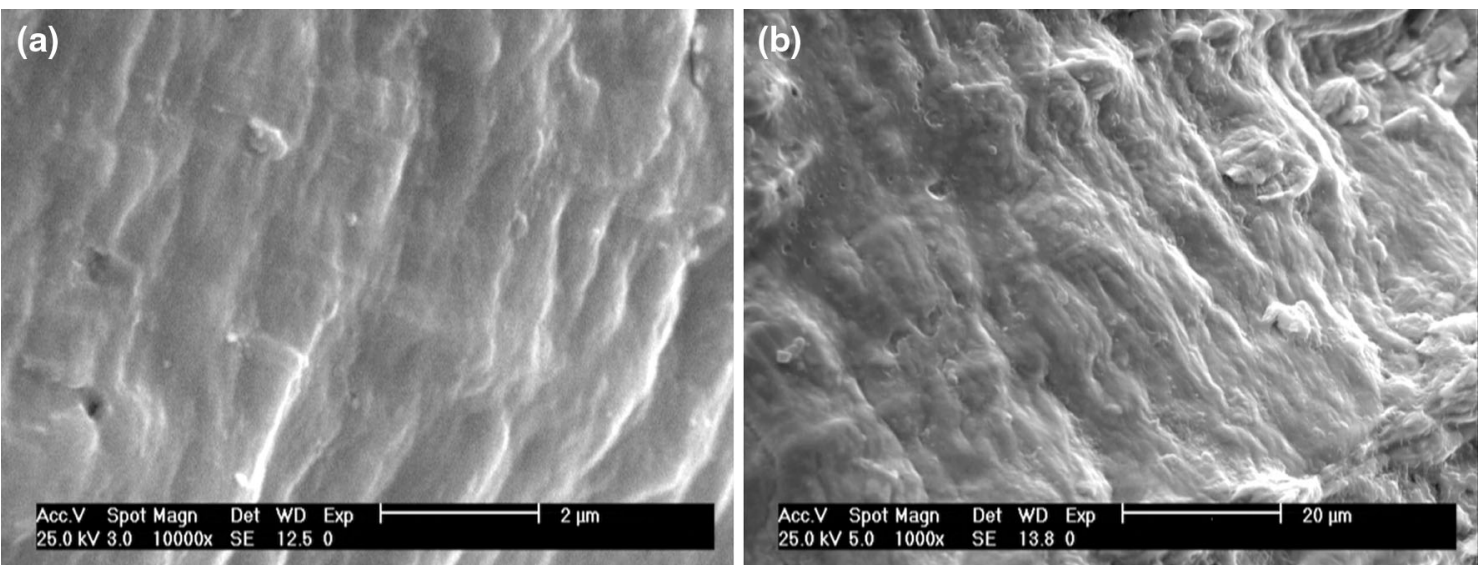

Fig. 4 Fatigue striations formed in nanostructured sample a, coarse-grain sample b
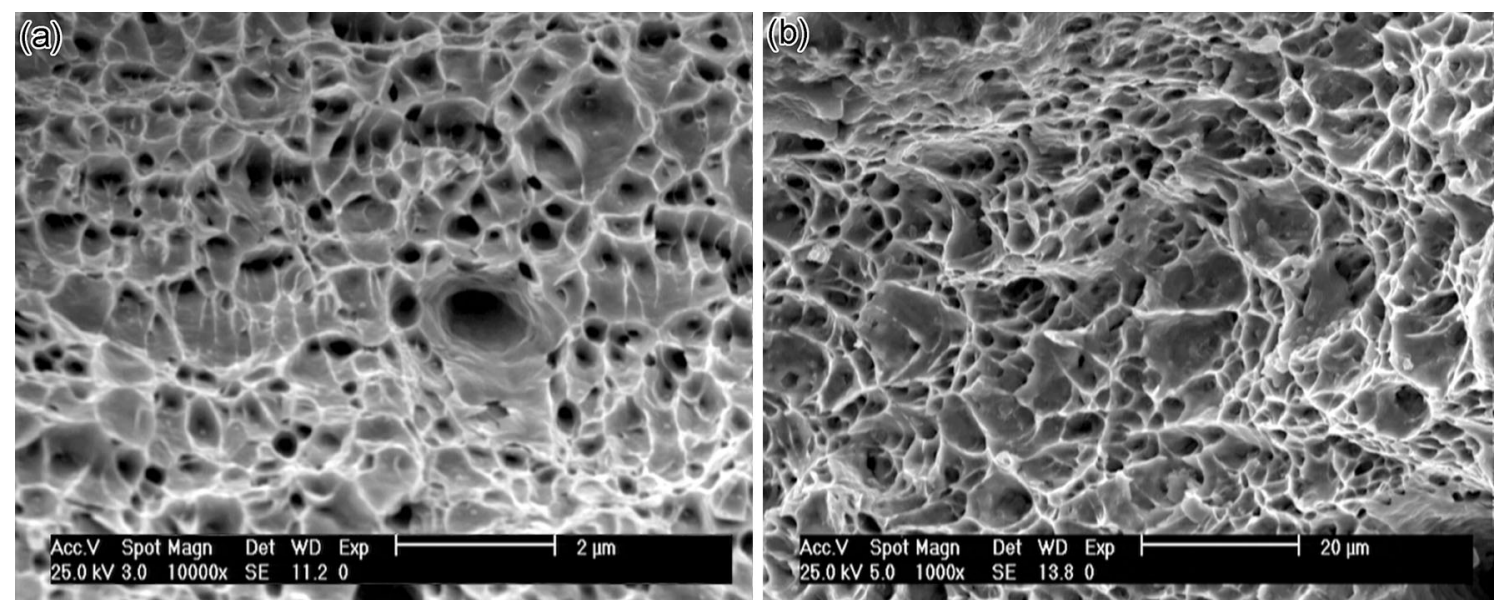

Fig. 5 The dimples formed in nanostructured sample a, coarse-grain sample $\mathbf{b}$ 
formed by FSP. Therefore, in terms of fatigue resistance, the smaller the grain size, the higher the hardness will be. Besides, more than threefold increase in the hardness of nanostructure surface can be of great importance in terms of fatigue strength. That is because the initiation of fatigue crack depends strongly on the hardness/strength and the grain size of surface layer.

Figure 3 compares the S-N curves regarding the initial coarse-grain and nanograin IF steel produced by FSP. In case of nanostructured case, the samples were machined completely from the stirred zone. Obviously, the fatigue life of FSPed sample is about $50 \%$ higher than that of the sample with initial coarse structure. With decreasing the grains to nanosize, the dislocation activity is significantly reduced. Consequently, the slip, as the main prerequisite for formation of intrusions and extrusions, is restricted remarkably. In such a case, the crack nucleation is highly restrained. Besides, the smaller the grains, the higher the volume fraction of grain boundaries will be. In case of nanograins, the high volume fraction of grain boundaries can effectively prevent dislocation sliding and inhibit crack nucleation. This corresponds to the dislocation model of cracks nucleation [20].

In case of specimen with a nanostructure layer, not only the crack is initiated with a prolonged delay, but also the coarse-grain structure in the center of specimen slows down the crack growth rate. Therefore, the fatigue life can significantly increase due to enhanced resistance to both crack initiation and crack propagation. Huang et al. [21] and Wang et al. [22] reported the similar enhancement in the fatigue strength of nanocrystalline steels and iron produced via surface mechanical attrition treatment.

The SEMs taken from the fractured coarse-grain and nanograin specimens are shown in Figs. 4 and 5, respectively. In Fig. 4, the striations formed during the fatigue crack propagation are compared for both cases. It is of interest that in case of nanostructure samples, the striations spacing is also nanometric comparing to their micrometric size in case of coarse-grain structure. In case of former, the striations spacing ranges within 100-500 nm (Fig. 4a), whereas it changes from 1 to $4 \mu \mathrm{m}$ in case of latter (Fig. 4b).

As for the final fracture stage, the nanodimples (Fig. 5a) are formed in nanostructured samples comparing to the microdimples (Fig. 5b) regarding the coarse-structure case. Besides, the nanodimples are equiaxed as well as more uniform and homogenous than microdimples.

\subsection{Corrosion Behavior}

Figure 6 illustrates $E_{\text {corr }}$ versus $\log i_{\text {corr }}$ plots (where $E_{\text {corr }}$ is the potential, and $i_{\text {corr }}$ is the density of corrosion current) of both nanograin and coarse-grain IF steel obtained at
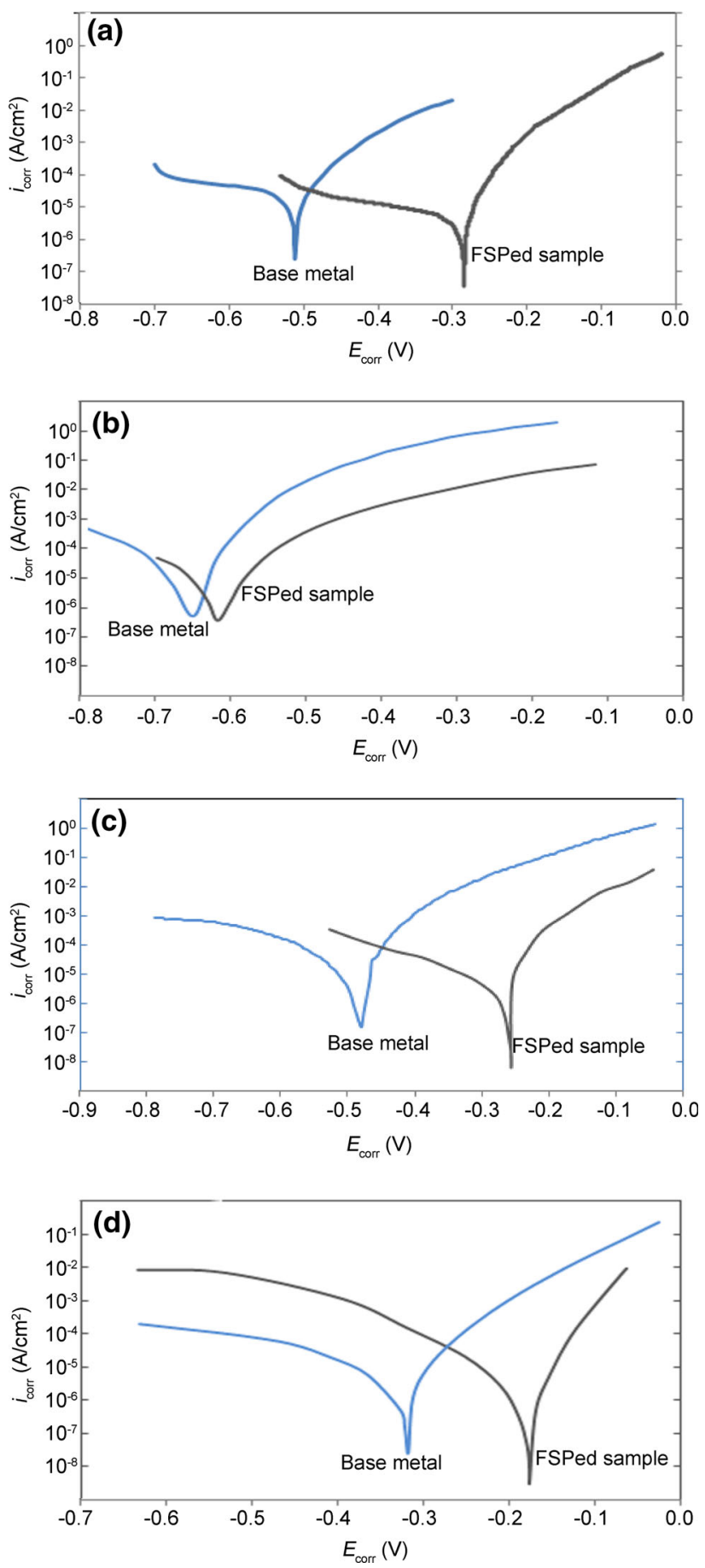

Fig. 6 potentiodynamic polarization curves for the coarse-grain and nano grain IF steel in different solutions: a $3.5 \mathrm{wt} \% \mathrm{NaCl}$, b $0.1 \mathrm{~mol} / \mathrm{L}$ $\mathrm{H}_{2} \mathrm{SO}_{4}$, c $2.5 \mathrm{wt} \% \mathrm{NaCl}+0.05 \mathrm{~mol} / \mathrm{L} \mathrm{H}_{2} \mathrm{SO}_{4}$, d $3.5 \mathrm{wt} \% \mathrm{NaCl}+$ $0.1 \mathrm{~mol} / \mathrm{L} \mathrm{H}_{2} \mathrm{SO}_{4}$

different corrosive media. The $i_{\text {corr }}$ and $E_{\text {corr }}$ values obtained from all conditions are summarized in Table 1. Using Tafel extrapolation method, $i_{\text {corr }}$ and $E_{\text {corr }}$ can be extracted from $E_{\text {corr }}$ versus $\log i_{\text {corr }}$ plots presented in Fig. 6. 
Table 1 Corrosion parameters of nanograin and coarse-grain IF steels in different corrosive media

\begin{tabular}{|c|c|c|c|c|}
\hline \multirow[t]{2}{*}{ Solution } & \multicolumn{2}{|l|}{$E_{\text {corr }}(\mathrm{V})$} & \multicolumn{2}{|l|}{$i_{\text {corr }}\left(\mathrm{A} / \mathrm{cm}^{2}\right)$} \\
\hline & CG IF steel & NG IF steel & CG IF steel & NG IF steel \\
\hline $3.5 \mathrm{wt} \% \mathrm{NaCl}$ & -0.59 & -0.28 & $5.34 \times 10^{-6}$ & $1.69 \times 10^{-7}$ \\
\hline $0.1 \mathrm{~mol} \mathrm{H}_{2} \mathrm{SO}_{4}$ & -0.67 & -0.61 & $6.43 \times 10^{-5}$ & $2.55 \times 10^{-5}$ \\
\hline $2.5 \mathrm{wt} \% \mathrm{NaCl}+0.05 \mathrm{~mol} / \mathrm{L} \mathrm{H}_{2} \mathrm{SO}_{4}$ & -0.48 & -0.26 & $5.78 \times 10^{-6}$ & $2.88 \times 10^{-8}$ \\
\hline $3.5 \mathrm{wt} \% \mathrm{NaCl}+0.1 \mathrm{~mol} / \mathrm{L} \mathrm{H}_{2} \mathrm{SO}_{4}$ & -0.32 & -0.18 & $5.72 \times 10^{-7}$ & $2.22 \times 10^{-8}$ \\
\hline
\end{tabular}
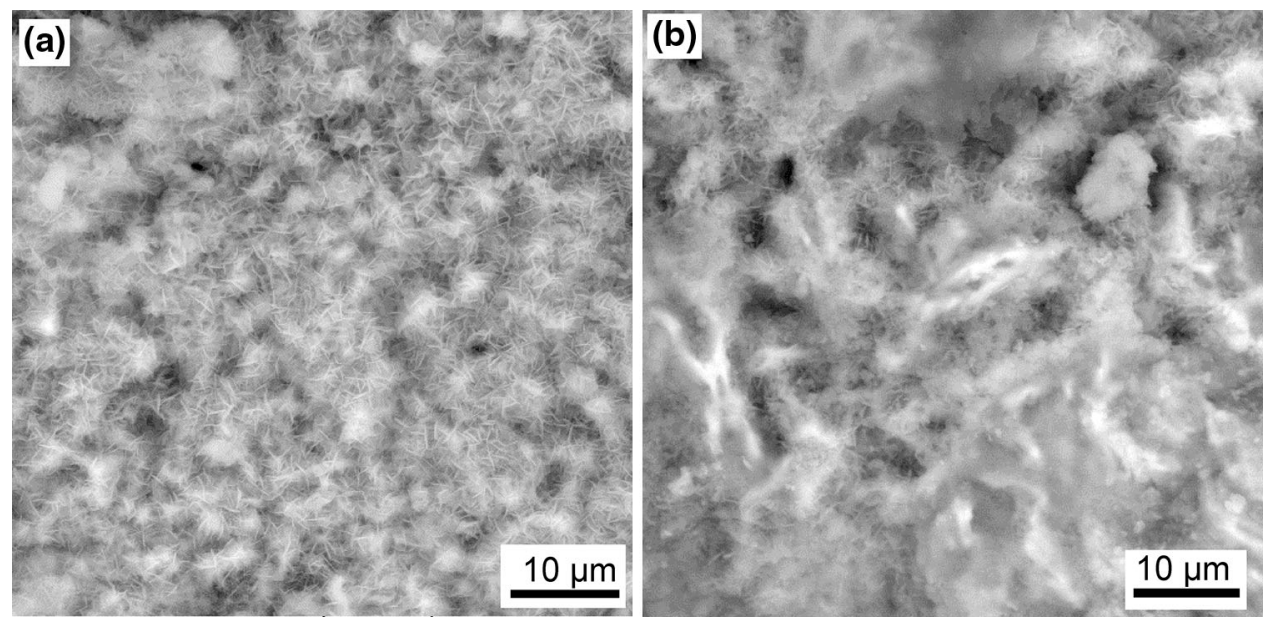

Fig. 7 SEMs of nano grain a coarse-grain b IF steels exposed to $3.5 \mathrm{wt} \% \mathrm{NaCl}$ medium

It is observed that the corrosion rate $\left(i_{\text {corr }}\right)$ of the base metal with the grain size of $60 \mu \mathrm{m}$ is considerably higher than that of its FSPed counterpart. Consequently, it is believed that the nanograin structure promotes the passivity and exhibits higher corrosion resistance. The present results are in good agreement with previous studies reporting that the corrosion resistance increases with decreasing the grain size to nanograins [19, 23-25].

Figure 7 compares the SEMs of coarse-grain and nanograin IF steel exposed to $3.5 \mathrm{wt} \% \mathrm{NaCl}$ medium. Accordingly, comparing to the coarse-grain specimen, the corrosion products in case of nanograin specimens are finely dispersed and more homogenous having no extensive localized pitting.

Figure 8 illustrates the SEMs of coarse-grain and nanograin IF steel exposed to $0.1 \mathrm{~mol} / \mathrm{L} \mathrm{H}_{2} \mathrm{SO}_{4}$ solution. As it is depicted, severe intergranular cracking was occured in the coarse-grain specimen, while a uniform passive layer was formed in nanograin specimen. This can be attributed to the amorphous-like structure of grain boundaries in nanocrystalline specimen, which results in higher corrosion resistance.

Considering the SEM images of coarse-grain and nanograin IF steel subjected to $3.5 \mathrm{wt} \% \mathrm{NaCl}+0.1 \mathrm{~mol}$ $\mathrm{H}_{2} \mathrm{SO}_{4}$ solution (Fig. 9), it is clear that the damage received to the nanograin sample is less severe than the coarse-grain case.

The results of potentiodynamic polarization curves (Fig. 6) reveal the enhancement of the corrosion current density $\left(i_{\text {corr }}\right)$ by changing the corrosion medium from the salt-containing solution (No. 1) to the acidic one (No. 2).

Furthermore, the corrosion resistance of $\mathrm{H}_{2} \mathrm{SO}_{4}$-containing solutions increased by adding $\mathrm{NaCl}$ into the bath, which can be attributed to the improvement in the passive film formation. In other words, the addition of $\mathrm{NaCl}$ to the $\mathrm{H}_{2} \mathrm{SO}_{4}$ medium appears to decrease the corrosion current density. This is an interesting finding and suggests that $\mathrm{NaCl}$ can act as a corrosion inhibitor in $\mathrm{H}_{2} \mathrm{SO}_{4}$-containing solutions. This is also of great importance in terms of industrial applications. That is because during pickling, known as a surface treatment process, the sulfuric acid is used to remove the mill scales (composed of iron oxides) from rolled sheets. Therefore, the addition of proper amount of $\mathrm{NaCl}$ to the pickling solution can enhance the corrosion resistance of sheets as well.

Besides, excessive metal loss by the chemical reaction during acid pickling results in poor surface quality of sheets. In such cases, adding $\mathrm{NaCl}$ to the sulfuric acid of pickling baths can minimize the metal loss and promote the surface quality of sheets. In the case of IF steel, it has been 

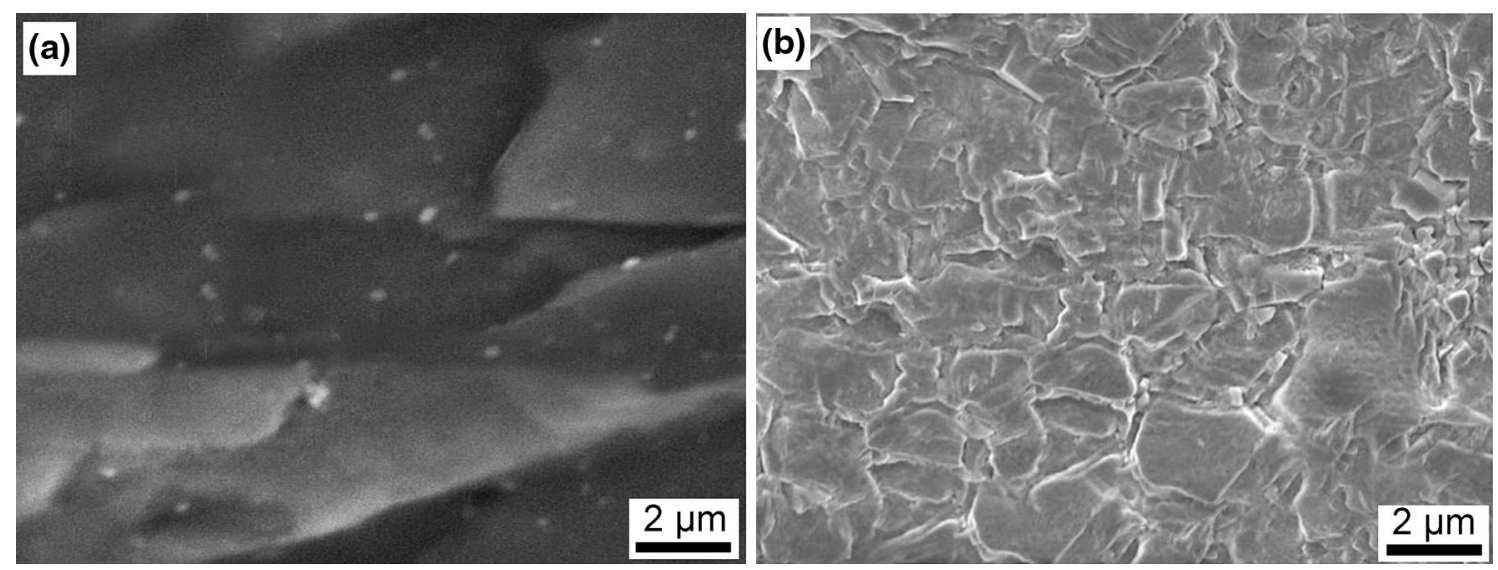

Fig. 8 SEMs of coarse-grain a nanograin b IF steels exposed to $0.1 \mathrm{~mol} / \mathrm{L} \mathrm{H}_{2} \mathrm{SO}_{4}$ medium
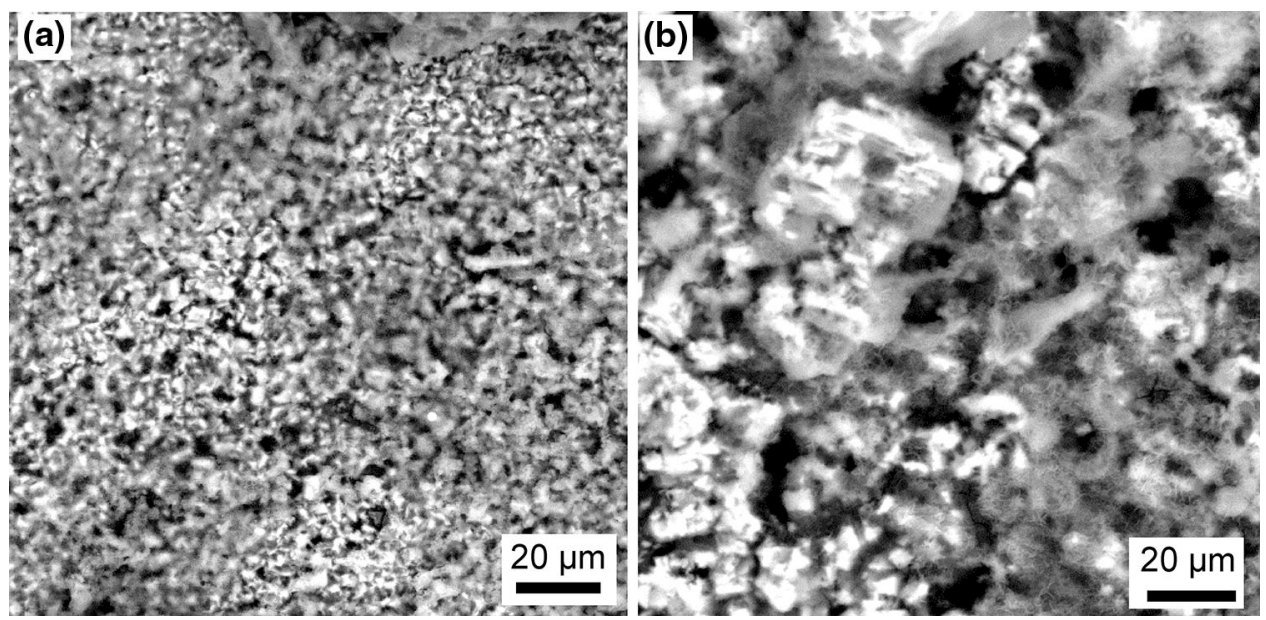

Fig. 9 SEMs of nanograin a coarse-grain b IF steels exposed to $3.5 \mathrm{wt} \% \mathrm{NaCl}+0.1 \mathrm{~mol} / \mathrm{L} \mathrm{H}_{2} \mathrm{SO}_{4}$ medium

reported [22] that the following reaction usually takes place at anodic areas:

$\mathrm{Fe} \rightarrow \mathrm{Fe}^{2+}+2 \mathrm{e}$.

While the oxygen reduction according to the cathodic reaction is:

$\mathrm{O}_{2}+2 \mathrm{H}_{2} \mathrm{O}+4 \mathrm{e} \rightarrow 4 \mathrm{OH}^{-}$.

In a broad sense, the corrosion of steel results in the formation of ferric oxides and ferrous hydroxide such as $\mathrm{Fe}_{3} \mathrm{O}_{4}, \mathrm{Fe}_{2} \mathrm{O}_{3}, \mathrm{Fe}(\mathrm{OH})$ and $\mathrm{FeOOH}$, which composes the diffusion-barrier layer next to the iron surface through which the oxygen must diffuse. Among the mentioned compounds, the $\mathrm{FeO}$ and $\mathrm{FeOOH}$ form a more adherent layer than $\mathrm{Fe}_{3} \mathrm{O}_{4}$ and $\mathrm{Fe}_{2} \mathrm{O}_{3}$, thereby tend to minimize the corrosive attack.

In the acidic media, the adsorbed $\mathrm{Fe}(\mathrm{OH})_{\mathrm{ad}}$ species can also be formed in accordance with the following reaction [24]:
$\mathrm{Fe}+\mathrm{OH}^{-} \Leftrightarrow \mathrm{Fe}(\mathrm{OH})_{\mathrm{ad}}+\mathrm{e}$.

The formation of passive films can be promoted by the absorption of corrosion products such as $\mathrm{Fe}(\mathrm{OH})_{\mathrm{ad}}$ at the kink sites presented on the surface of materials. It can be explained by the fact that the kinks existed on the surface of metals are most likely to act as the preferential and heterogeneous nucleation sites for the adsorption of corrosion product(s) [26].

Regarding the FSPed IF steel, the density of kink sites can be remarkably higher than untreated steel, owing to the severe plastic deformation applied on the surface. As a result, a more uniform and smooth passive film can be formed on the surface of FSPed steel comparing to that of coarse-grain steel. In case of latter, the passive layer is localized and non-uniform.

On the other hand, it is believed that because of high volume fraction of grain boundaries in the nanostructured material, the diffusivity of elements can be much higher so as to promote the formation of dense passive films. Thus, 
the higher corrosion resistance of nanograin sample can be due to the combination of extra heterogeneous nucleation sites for formation of passive film and the enhanced diffusivity.

\section{Conclusions}

A nanograin layer of about $150 \mu \mathrm{m}$ thick was formed on the surface of an IF steel using FSP technique. Comparing to coarse-grain IF steel, the formation of nanograins resulted in about $50 \%$ increase in the fatigue strength of nanostructured IF steel. Surface nanostructuring can be a unique approach to enhance the fatigue resistance of materials, especially for IF steels used widely as car bodies. The formation of nanospacing striations and nanodimples was the characteristics of nanograin IF steel after fatigue failure. The highest corrosion resistance belongs to the nanostructured steel due to the combination of extra/heterogeneous nucleation sites and the enhanced diffusivity leading to the formation of dense passive films.

\section{References}

[1] L. Shaw, J.W. Tian, A.L. Ortiz, K. Dai, J.C. Villegas, P.K. Liaw, R. Ren, D.L. Klarstrom, Mater. Sci. Eng. A 527, 986 (2010)

[2] L. Du, S. Yao, J. Hu, H. Lan, H. Xie, G. Wang, Acta Metall. Sin. (Engl. Lett.) 27, 508 (2014)

[3] G. Liu, S.C. Wang, X.F. Lou, J. Lu, K. Lu, Scr. Mater. 44, 1792 (2001)

[4] N.R. Tao, Z.B. Wang, W.P. Tong, M.L. Sui, J. Lu, K. Lu, Acta Mater. 50, 4603 (2002)

[5] J. Villegas, K. Dai, L. Shaw, T.S. Srivatsan, R. Varin (eds.), in Processing and Fabrication of Advanced Materials, (ASM International, Materials Park, Ohio, 2007), p. 358
[6] L. Shaw, C. Suryanarayana, R. Mishra (eds.), in Processing and Properties of Structural Nanomaterials, (TMS, Warrendale, PA, 2003), p. 61

[7] K. Dehghani, M. Nasirzadeh, S. Bagherzadeh, J. Mater. 224, 190 (2010)

[8] C.I. Chang, X.H. Du, J.C. Huang, Scr. Mater 59, 356 (2008)

[9] A. Yazdipour, A. Shafiei, M.K. Dehghani, Mater. Sci. Eng. A 527, 192 (2009)

[10] L. Karthikeyan, V.S. Senthilkumar, V. Balasubramanian, S. Natarajan, Mater. Des. 30, 2237 (2009)

[11] B. Zahmatkesh, M.H. Enayati, F. Karimzadeh, Mater. Des. 31, 4891 (2010)

[12] A. Chabok, K. Dehghani, Mater. Sci. Eng. A 528, 309 (2010)

[13] X.Y. Zhang, M.H. Shi, C. Li, N.F. Liu, Y.M. Wei, Mater. Sci. Eng. A 448, 259 (2007)

[14] B. Hadzima, M. Janecek, Y. Estrin, H.S. Kim, Mater. Sci. Eng. A 462, 243 (2007)

[15] X.Y. Mao, D.Y. Li, F. Fang, R.S. Tan, J.Q. Jiang, Mater. Sci. Eng. A 527, 2875 (2010)

[16] H. Lee, D. Kim, J. Jung, Y. Pyoun, K. Shin, Corros. Sci. 51, 2826 (2009)

[17] A. Bahari, S.A. Babanezhad, F. Ashrafi, M. Moshahedi, World Appl. Sci. J. 7, 22 (2009)

[18] A. Balyanov, J. Kutnyakova, N.A. Amirkhanova, V.V. Stolyarov, R.Z. Valiev, X.Z. Liao, Y.H. Zhao, Y.B. Jiang, X.F. Xu, T.C. Lowe, Y.T. Zhu, Scr. Mater 51, 225 (2004)

[19] K. Dehghani, M. Hosseini, A. Nekahi, Int. J. Mater. Res. 104, 999 (2013)

[20] K. Tanaka, T. Mura, J. Appl. Mech. 48, 97 (1981)

[21] H.W. Huang, Z.B. Wang, X.P. Yong, K. Lu, Mater. Sci. Technol. 29, 1200 (2013)

[22] Z.B. Wang, N.R. Tao, W.P. Tong, J. Lv, K. Lu, Acta Mater. 51, 4319 (2003)

[23] T.P. Hoar, J. Electrochem. Soc. 117, 17C (1970)

[24] M.P. Ryan, R.C. Newman, G.E. Thompson, Philos. Mag. B 70, 241 (1994)

[25] M.P. Ryan, S. Fugimoto, G.E. Thompson, R.C. Newman, Mater. Sci. Forum 185, 233 (1995)

[26] Metals Handbook, vol. 13A, (ASM Intnational, Materials Park, OH, USA, 2003) 